Cinémas

Revue d'études cinématographiques

Journal of Film Studies

\title{
French Cancan et le spectateur mobile
}

\section{Édouard Arnoldy}

Volume 12, numéro 3, printemps 2002

Cinélekta 4

URI : https://id.erudit.org/iderudit/000733ar

DOI : https://doi.org/10.7202/000733ar

Aller au sommaire du numéro

Éditeur(s)

Cinémas

ISSN

1181-6945 (imprimé)

1705-6500 (numérique)

Découvrir la revue

Citer cet article

Arnoldy, É. (2002). French Cancan et le spectateur mobile. Cinémas, 12(3),

11-31. https://doi.org/10.7202/000733ar
Résumé de l'article

L'objectif de cet article est d'inscrire French Cancan (Jean Renoir, 1955) dans le cadre d'une réflexion consacrée au regard des spectateurs. Ce texte, qui a pour double objet le mouvement et le regard, considère précisément que le mouvement du regard du personnage pivot de ce film (Danglard-Gabin) sert le rythme d'une comédie musicale plutôt caractérisée par une apparente impassibilité de la caméra. Hymne à la peinture impressionniste, French Cancan n'en constituerait pas moins selon l'auteur un bel hommage au Cinématographe, par l'attention qu'il porte aux mouvements dans l'image. 


\title{
French Cancan et le spectateur mobile
}

\section{Édouard Arnoldy}

\begin{abstract}
RÉSUMÉ
L'objectif de cet article est d'inscrire French Cancan (Jean Renoir, 1955) dans le cadre d'une réflexion consacrée au regard des spectateurs. Ce texte, qui a pour double objet le mouvement et le regard, considère pré cisément que le mouvement du regard du personnage pivot de ce film ( $D$ anglard-G abin) sert le rythme d'une comédie musicale plutôt caractérisée par une apparente impassibilité de la caméra. H ymne à la peinture impressionniste, French Cancan n'en constituerait pas moins selon l'auteur un bel hommage au Cinématographe, par l'attention qu'il porte aux mouvements dans l'image.
\end{abstract}

\section{ABST RACT}

The goal of this article is to inscribe Jean Renoir's film French Cancan (1955) in the framework of an analytical model dedicated to the spectator's gaze. This article, whose dual focus is movement and the gaze, considers the movement of the gaze of the film's principal character ( $D$ anglard-Gabin) precisely as one suited to the rhythm of the musical comedy, one of whose characteristics is the apparent passivity of the camera. A hymn to Impressionist painting, French Cancan was also, according to its director, no less an homage to the ciné matographe, by conferring a privileged position upon movement within the image. 


\section{«Projections parlantes», talking shorts, comédies musicales et regards aux spectateurs}

De l'éclosion des premiers cafés-concerts dans Paris au XIXe siècle à la danse finale de French Cancan (1955) de Jean Renoir, voilà un parcours de l'histoire du cinéma (parlant) qui mériterait certainement d'être effectué. De l'édification d'estrades dans des cabarets, au filmage de chansons de cabarets célèbres à l'aube des années dix (par exemple les «projections parlantes» ou «phonoscènes» G aumont) et à l'implosion de la scène dans l'épilogue de French Cancan, en passant par les «scènes chantées» et la comédie musicale de Jean Renoir, ainsi pourrait s'effectuer ce parcours assez singulier, telle une rotation de trois cent soixante degrés autour du regard dans les «films chantants et parlants». Cette rotation complèterait un trajet au cœur d'une histoire du cinéma parlant qui aurait pour souci de dire avec insistance qu'une histoire du cinéma sonore et parlant croise, en plusieurs de ses incidences, une histoire du regard et des images en mouvement.

De la mise sur scène des chanteurs offerts au regard de tous les spectateurs des cafés-concerts à la mise en scène du regard spectatoriel dans le film de Jean Renoir, il s'agirait de dire que I'œil et l'oreille participent - de très près et dans un même élan - au glissement irrévocable du film muet au cinéma parlant ${ }^{1}$. Allant un peu à contre-courant de ce lieu commun de l'histoire du cinéma qui maintient une stricte filiation entre le regard au spectateur dans les théâtres ou les cafés-concerts et le regardcaméra du cinéma, ce glissement aurait plutôt tendance à dire que le regard à la caméra pourrait n'avoir que des rapports assez lâches avec une certaine forme de frontalité qui, effectivement, prévaut largement au caféconcert ou au music-hall, et parfois au théâtre. C'est essentiellement cette hypothèse qui sera examinée dans les pages qui suivent. Du moins s'agira-t-il de regarder autrement, soit par l'autre bout de la lorgnette, cette hypothétique filiation, pour interroger cette correspondance d'un regard (non cinématographique) à l'autre (filmique).

À partir de ce réseau de relations entre le cinéma et certaines formes particulières de spectacle comme le music-hall ou le café 
concert, il y a d'abord lieu de ne pas confondre I'histoire de la forme filmique et I'histoire du cinéma ni de les amalgamer de façon décousue et inconsidérée. La distinction entre les deux histoires - replacée dans un cadre plus large - paraît essentielle, dans la mesure où l'une est trop souvent privilégiée aux dépens de l'autre, au point de les mettre de force l'une en face de l'autre, voire de les rendre inconciliables. D 'une manière générale, I'histoire de la forme filmique se plaît-elle sans doute trop souvent à enquêter sur les déterminations et l'évolution de la forme du film à travers le temps, dans la perspective d'un enchaînement passablement prévisible et ininterrompu d'événements. D e plus, dans une telle perspective, on est trop souvent enclin à évaluer, tant sur un axe prospectif que rétrospectif, le cinéma d'après une hypothétique forme filmique classique. Enfin, sans dire que l'histoire de la forme filmique n'est pas de I'histoire du cinéma, le risque est grand pour une histoire de la forme filmique de mal évaluer les attributs que le cinéma partage d'aventure avec d'autres disciplines et, par voie de conséquence, d'interpréter à mauvais escient telle ou telle particularité stylistique d'un film 2 ${ }^{2}$. À l'inverse, l'histoire du cinéma a parfois tendance - sous le couvert d'une louable rigueur - à faire l'économie des films et de l'esthétique, et à préférer certains documents (le «non-film ») à d'autres (le film). Pourtant, il n'y a rien d'intempestif à dire que l'histoire du cinéma, c'est aussi une histoire de films, de figures, de formes. Le présent article, plutôt guidé par un sens certain du compromis, se situe aux frontières mouvantes des histoires du cinéma et de la forme filmique.

Pour en revenir un instant à d'éventuels rapports particuliers entre le cinéma et le théâtre ou les spectacles, sans doute ces rapports reposent-ils souvent sur des malentendus et sur une vision parcellaire de l'art dramatique et du cinéma(tographe). En repensant à Frank Kessler et à Sabine Lenk (1997, p. 185), par ricochet, il faut convenir que «l'opposition entre "cinématographicité" et "théâtralité" est au fond un faux problème, car il repose sur des conceptions beaucoup trop monolithiques à la fois du cinéma et de la scène ${ }^{3} »$. Faute d'espace, une telle problé matique sera juste effleurée en ces pages. Ce dont il sera plus Ionguement traité, c'est du regard assez singulier d'un personnage 
d'une comédie musicale française tournée au milieu des années cinquante. Ce personnage insolite officiera en quelque sorte en tant que guide des à-côtés d'une histoire du cinéma parlant dont l'ambition serait de s'intéresser aux ressemblances déplacées - selon une expression de Georges Didi-H uberman à propos de tout autre chose - entre les «projections parlantes», les scè nes ou spectacles filmés des années dix, les talking shorts de la fin des années vingt et les comédies musicales du cinéma parlant. C'est en ce sens que la frontalité des «scènes chantées» des années dix et des talking shorts ou des premiers talkies de la fin des années vingt mérite d'être regardée d'un œil attentif.

Sans revenir dans le détail sur la mise en scène des «phonoscènes » tournées dans les laboratoires G aumont vers 1906, peut-être est-il bon de rappeler que nombre des «projections parlantes» des années dix se caractérisent certainement par une frontalité ostensible: un chanteur, le plus souvent une vedette des cafés-concerts parisiens ou de l'opéra, est face à la caméra pour interpréter une chanson de son répertoire ou un extrait d'un livret renommé4. Souvent présentées en un plan, ces courtes scènes se clôturent généralement par un salut du chanteur adressé au public (situé dans une zone mal précisée, entre le hors-champ et le hors-cadre). Ces vues, parfois projetées dans des salles de cinéma récemment édifiées, souvent présentées dans des cabarets ou des foires, se résument communément à enregistrer sur une pellicule et un cylindre la mise sur scène des étoiles des cafés-concerts et de l'opéra ou de l'opérette. Lorsque, à la fin des années vingt, les frères Warner (et d'autres producteurs) mettent à l'ordre du jour le «film sonore», il est à nouveau question de présenter des vedettes populaires aux spectateurs des salles de cinéma - en l'occurrence des stars de l'opéra et de Broadway - en guise d'attraction ${ }^{5}$. Au fond, pas plus qu'au début des années dix, il n'est alors question de dresser «le» cinéma parlant contre «le» cinéma muet.

Par contre, il s'agit bien de proposer des «films chantants et parlants» qui viendraient s'ajouter aux programmes, avec pour ambition de compléter ou de parfaire ces soirées, et de les distinguer de l'offre des salles et des compagnies rivales. Avec le temps, ces bandes prendront la place des spectacles en direct, 
pour réserver les séances de cinéma aux seuls films. Le plus frappant, à première vue du moins, est indéniablement que les films proposés par la Warner au début de sa collaboration avec la Vitaphone Corporation ressemblent étrangement aux scènes produites par $\mathrm{G}$ aumont vingt ans plus tôt. Le répertoire des uns et des autres concilie des genres de noblesse culturelle variable. Comme les «phono-scènes», les talking shorts se singularisent eux aussi par une frontalité criante, ou, si l'on veut, par un recours systématique au regard à la caméra ${ }^{6}$. À l'analyse cependant, ces analogies et cette filiation entre les films des années dix et vingt - presque péremptoires - prêtent flanc à la critique peu à peu. De légères dissemblances entre les films de ces deux époques augurent certainement d'un rapport autre au regard, en train de prendre corps dans les «films chantants et parlants» des années vingt. De la mise sur scène des chanteurs à la mise en scène de spectacles chantés au cinéma, du regard au spectateur des spectacles filmés au regard au spectateur du cinéma (parlant), sans doute de telles dissemblances présagent-elles, à la fin des années vingt, de la transition insensible des «scènes chantées» au «film chantant et parlant».

\section{D u mouvement et des regards dans les vaudfilms et les talking shorts}

De prime abord, dans la production Warner Vitaphone, le salut du chanteur et, plus globalement, la frontalité relient les uns aux autres une grande part de ces «films», puis constituent les indices indéfectibles de la complicité qui semble lier le chanteur au spectateur de cinéma. Les façons de s'adresser au public n'en sont pas moins sensiblement différentes dans nombre des talking shorts. Des films comme Betwen the Acts at the 0 pera (1926), Twinkle, Twinkle (1927) ou Lambchops (1929), où les vedettes s'adressent directement au public, permettent de mieux comprendre certains écarts, à la réflexion pas si insensibles que cela, dans les rapports entre le chanteur et le spectateur de cinéma ${ }^{7}$. D ans Between the Acts at the 0 pera, Willie et Eugene $\mathrm{H}$ oward sortent de l'opéra pour s'entretenir face à la caméra. Les deux frères, I'un coiffé d'un chapeau haut de forme, l'autre d'un canotier blanc, dialoguent sur un ton léger et bon enfant, pour 
finir par un numéro de chant et de danse. Pour clôturer leur numéro et la «scène filmée», ils saluent leur public en s'inclinant, le chapeau bas. Relativement épargnée par la critique à sa sortie en 1926, la «scène chantée et parlée» n'en sera pas moins égratignée par la suite, semblet-il pour sa forte connivence avec les attractions chantées des années antérieures et ses trop lâches affinités avec le «film chantant et parlant » en vogue.

En effet, depuis un ou deux ans, la critique - qui se fait l'écho des projections dans les villes de province - ne manque pas de déconsidérer quelques-uns des premiers Vitaphone shorts. Les journalistes n'hésitent pas à décrier la pauvreté récurrente de la mise en scène de certains des talking shorts. Ainsi, un correspondant de Variety (anonyme, 1928, p. 14 - notre traduction) à Clinton dans l'État de N ew York considèret-il en juin 1928 à propos de Between the Acts at the 0 pera que cette comedy talk and song risque de compromettre la bonne réputation des frères H oward, car «les numéros musicaux du finale sont inadéquats. Ils discréditent injustement les deux garçons auprès d'un public qui ne les connaît pas. [... ] Tout porte à croire que dans un registre approprié, les frères $\mathrm{H}$ oward devraient connaître plus de réussite avec les films parlants». Par contre, plusieurs de ces films talk and song connaissent un accueil assez favorable. Sans doute les critiques de cinéma, qui reprochent alors à $\mathrm{Al}$ Jolson d'être un chanteur et non un acteur, sont-ils particulièrement sensibles au fait que ces numéros (parlés et chantés) ont revêtu peu à peu les atours de films (parlés et chantés). Parce que les critiques sont en porteà-faux entre un média et un médium, à circuler ainsi entre des pratiques diverses, le passage de l'un à l'autre est à tout le moins hésitant, incertain.

D ans le même registre, Twinkle, Twinkle est une comédie de vaudeville à grand succès, mettant en vedette Joe $E$. Brown et Perquita Courtney. In the M ovies, le second titre de Twinkle, Twinkle, résume l'essentiel de son scénario. Au cours d'une pause dans le tournage d'un film muet, un homme se présente, une carte de visite en main, à l'actrice vedette du film. II tente de convaincre cette dernière de ses dons d'acteur; elle lui propose de jouer l'angoisse, l'indignation (pas l'indigestion, lui ditelle, après un «léger malentendu»), la joie, l'amour, etc. La pré 
sence de l'inconnu sur le plateau constitue le prétexte narratif à la mise en route d'un numéro de vaudeville privilégiant les jeux de mots et les expressions corporelles. À la fin de ce numéro, l'actrice de cinéma s'en va. L'acteur en herbe la rappelle, lui disant qu'elle oublie son public, de fait dédaigné jusqu'alors par la star du muet. C'est bien Joe E. Brown, et non le personnage [de] in the movie[s], qui prend Perquita Courtney par la main: les comédiens de vaudeville saluent le public. Longtemps négligés par les acteurs dans Twinkle, Twinkle, à la dernière minute, les spectateurs de cinéma ne sont heureusement pas oubliés par les comédiens de vaudeville.

À l'inverse, Lambchops, attribué à M urray Roth, ne s'encombre pas d'un tel détour narratif pour amener le numéro de chant et de danse du couple vedette. À peine apparus à l'écran (dans un salon bourgeois), George Burns et Grace Allen y vont d'un spontané «hello everybody», pour ensuite se présenter l'un à l'autre et entamer un long dialogue. Sans plus trop se préoccuper de la caméra, Burns et Allen chantent et dansent. La fin de ce petit film (en sept plans) est assez inédite. G race Allen regarde la caméra, avant de s'excuser; elle glisse quelques mots à l'oreille de son complice, puis rigole. Et lui de conclure à mots couverts: «T hat's the story. » 0 n n'en saura pas plus. G eorge Burns pousse Grace Allen dans le dos pour la diriger vers la sortie. Les deux vedettes s'en vont sur un air de musique, sans plus se retourner. Alors que le film prétend entretenir d'emblée une complicité franche entre les deux faces de l'écran ( «hello everybody »), I'ironie qui caractérise la scène finale marque pourtant une certaine distance entre le duo et les spectateurs du film, quand Grace Allen et George Burns quittent la scène. (Pour mieux entrer... in the movies?)

C'est bien là que se situe la particularité, ou plutôt l'ambiguïté de L ambchops. De prime abord entièrement articulé sur un numéro de music-hall, ce film dérape subrepticement jusqu'aux abords d'une narration. Son épilogue joue à plein d'un effet d'attente du narratif, lorsque les deux acteurs discutent entre eux. Il y a donc un décalage assez prononcé entre l'inaugural salut au public et la mise à l'écart de ce dernier lors de la discussion (chuchotée, inaudible) à la fin du film. L'effet d'attente du 
narratif est d'autant plus perceptible qu'il coïncide avec la frustration du spectateur, quand l'aparté entre les deux acteurs reste sans suite, puisque le film prend fin aussitôt après. Si le spectateur obtient, dans un premier temps, ce qu'il attend de Grace Allen et de G eorge Burns, c'est-à-dire un show donné par les deux vedettes, cet énigmatique finale le laisse dans une large mesure sur sa faim. D ans plusieurs films, précédant de peu ou contemporains de Lambchops, cet effet de distance entre spectateur de cinéma et vedette populaire s'obtient quand, précisément, l'apparente connivence entre le chanteur et son public paraît soulignée avec insistance par la présence de spectateurs dansle film.

$D$ ans cette vaste production d'époque, où s'inscrit fugitivement l'une ou l'autre des marques distinctives du spectacle filmé et du «film chanté et parlé», I dle Chatter (1929), mettant en vedette Lou H oltz, mérite une certaine attention. L'humour du film se fonde essentiellement sur une cascade de jeux de mots et une gestuelle vive. D epuis les premiers plans dans les loges jusqu'au salut final, I dle Chatter instaure un régime spectatoriel particulier. La complicité qui unit Lou H oltz et le spectateur va crescendo. I dle Chatter débute par le plan d'une malle déposée dans une loge, sur laquelle se trouve inscrit Lou H oltz, le nom du comédien en train de se maquiller et de se coiffer avant d'entrer en scène. Grâce à deux incursions rapides de la caméra dans la salle, on entraperçoit les spectacles qui s'y déroulent à ce moment-là (des girls de cabaret, puis un danseur et une danseuse acrobates). Devant son miroir, Lou H oltz se coiffe; il se lève, puis met son veston. Lorsqu'il sort de sa loge, tiré à quatre épingles, c'est pour, sans transition, entrer en scène. Face à la caméra, Lou H oltz se lance dans un long monologue où il ironise sur les ballets (qu'il dit «a-do-rer»). Entraînant son corps d'échalas d'un bout à l'autre de la scène, Lou Holtz fait mine d'imiter les pas de danse d'un ballet. Son one man show est interrompu: une voix, venue d'un hors-champ encore indistinct, l'interpelle pour lui demander le nom du spectacle qu'il est en train de railler.

Un véritable dialogue s'engage entre le comédien et son spectateur (interne). Lou $\mathrm{H}$ oltz fait mine de réfléchir, dit vaguement 
se souvenir d'un titre où il est question de fleurs. Le spectateur lui renvoie la balle et propose L e Barbier de Séville. À cet instant, Lou H oltz se tourne vers sa droite: un autre spectateur, lui aussi situé dans un hors-champ encore imprécis, suggère The Flying D utchman. Lou Holtz rejette cette proposition absurde, avant de dire qu'il s'agit en fait de Samson et Dalila. Sur cette note d'humour, le dialogue prend fin. Face à la caméra, Lou Holtz regarde droit devant lui, fier de son succès auprès d'un public hilare, avant de terminer son numéro en chanson. Le public applaudit à tout rompre. Après cette deuxième incursion du côté du public dans la salle de spectacle, le film (d'une vingtaine de plans) s'achève sur Lou H oltz saluant ses admirateurs.

De fait, dès qu'il est dans la salle, Lou Holtz ne quitte plus son public des yeux. Leur complicité va en augmentant, pour atteindre son point culminant lorsque $\mathrm{H}$ oltz dialogue avec certains spectateurs. Les applaudissements parachèvent une relation de sympathie qui, en rien, n'est factice. Par deux fois, les champs-contrechamps entre Lou H oltz et le public de la salle annulent définitivement la distance qui sépare la vedette de music-hall de son public. À l'exception des plans de coulisses ou depuis les coulisses (Lou H oltz dans sa loge, puis les girls et les danseurs acrobates), le point de vue privilégié est celui du spectateur de la salle de spectacle. Paradoxalement, cette bonne entente entre le spectateur et Lou H oltz paraît interdire tout rapprochement entre la vedette de music-hall et le spectateur du film Idle Chatter (ou toute identification du spectateur externe au spectateur interne). N éanmoins, le spectateur de cinéma paraît bénéficier, un bref instant, du privilège exclusif de péné trer dans les coulisses de la scène, jusque dans l'intimité de la loge de Lou Holtz. O r, d'une manière assez symptomatique, la star de Broadway ne lui prête jamais la moindre attention quand il est dans sa loge. Lou H oltz lui tourne presque systématique ment le dos (le plan de face dans la loge est en fait un plan de dos de la vedette qui se regarde dans un miroir), alors qu'à l'inverse, il fait toujours face au spectateur de la salle.

$D$ ans I dle Chatter, la frontalité - généralement admise comme l'indice indéfectible d'un rapport forcément direct et intime entre l'acteur ou le chanteur et le spectateur de cinéma - 
est insistante, mais n'en établit pas moins dans ce cas précis une distance franche entre le personnage à l'écran et son public des salles de cinéma, car ce n'est pas à ce dernier qu'il s'adresse directement mais bien à celui de la salle de spectacle. C'est, si l'on veut, tout le paradoxe de la mise en scène de ce film où Lou $\mathrm{H}$ oltz est presque invariablement face à la caméra alors que son regard (son show, ses bons mots, ses pas de danse) n'est pas directement adressé au spectateur de cinéma mais bien au spectateur dans la salle de spectacle ${ }^{8}$. D ans ce film singulier se perçoivent clairement les remous qui agitent la représentation ciné matographique, prise entre un regard-caméra plutôt transgressif quant à la narration cinématographique et un regard au spectateur plutôt dans la norme au music-hall (où la participation du public est souhaitée). En même temps que les liens entre Broadway et le cinéma parlant semblent par ailleurs se consolider, dans sa mise en forme, le «film chantant et parlant » paraît prendre une certaine distance avec son spectateur, à plus forte raison lorsque la vedette du film interpelle le spectateur du spectacle filmé. De plus en plus solitaire, de moins en moins solidaire, le spectateur des films participe de loin en loin aux spectacles filmés 9 .

Dans un même mouvement, le spectateur de cinéma - qui se trouve de la sorte mis à distance, voire presque à l'écart du spectacle - a accès aux coulisses de la scène, pour voir toujours davantage que le spectateur des salles de spectacle. D 'un côté, le spectateur ordinaire du cinéma parlant perd une large part de la complicité qui l'unit aux showmen. D ans le même temps, son regard se caractérise, et insiste de plus en plus, par sa mobilité, par sa liberté de mouvements. C'est peut-être un des paradoxes de ce cinéma suspendu entre le muet et le parlant: alors que l'époque est souvent décriée pour le statisme de ses films et le retour en grâce du "théâtre filmé», la caméra des talking shorts et des comédies musicales - autant de films qui ne dissimulent pas leurs emprunts à un répertoire populaire et leur recours au spectacle spectaculaire - paraît avoir pour prérogative d'accorder dès le départ une réelle mobilité au regard du spectateur du cinéma parlant. Autant dire que ces films soulignent avec force ce qui distingue en grande partie le regard du spectateur de spectacle 
en direct (thêâtre, music-hall, café-concert, etc.) et le regard du spectateur de cinéma (désormais parlant ${ }^{10}$ ).

\section{D u regard mobile d'un «spectateur » dans French C ancan}

C'est précisément de cette distinction entre le regard plutôt statique des spectateurs des cabarets, des music-halls ou des théâtres et les mouvements du regard spectatoriel au cinéma dont il est largement question dans un film qui a, non par hasard, pour cadre les cabarets du M ontmartre de la fin du $X I x^{e}$ siècle. Dans French Cancan (1955), Jean Renoir filme en effet au plus près les mouvements du regard spectatoriel, pour, sans conteste, accorder un rôle particulièrement déterminant à la mobilité du regard du spectateur de cinéma. M aîtrisant à la fois les histoires de la forme filmique et du cinéma, I'histoire de la peinture et des arts populaires de la scène, Jean Renoir met à l'épreuve, dans ce film en particulier, une pensée-cinéma modulée selon un axe cinéma-théâtrepeinture dont la roue serait le regard spectatoriel. D ans French Cancan, le regard spectatoriel est mobilisé par le personnage pivot interprété par Jean Gabin. Ayant pour toile de fond la vie nocturne parisienne du début du $x x^{e}$ siècle et certains moments de la vie romanesque du fondateur du M oulin-Rouge, French Cancan traque le passé - déjà presque effacé - du cinéma français, pour exécuter, par l'entremise de son personnage principal ( $D$ anglard-Gabin), un chassé croisé entre le cinéma, le café-concert et la peinture.

Confrontant French Cancan au M oulin de la Galette d'Auguste Renoir, André Bazin pense que, contrairement à leurs apparences trompeuses, ces deux œuvres sont «bien plus qu'un divertissement pictural sur un divertissement social ». Pour lui, Jean Renoir n'évoque jamais une époque de la peinture «par une imitation extérieure de ses caractéristiques formelles», car «sa mise en scène s'ordonne spontanément et naturellement en conformité de style avec cette peinture» (Bazin, 1971, p. 123-124). Exclusivement articulé autour de la profonde intimité qui unit, dans ce film, Renoir et son père, le propos d'André Bazin pourrait se résumer à cette phrase: «Renoir ne part jamais de la peinture, il y arrive. » Privilégiant le point de vue de Danglard, le film serait d'abord travaillé par ce mouvement, comme en de 
subtiles touches, du cinéma vers la peinture. Loin de l'œuvre au sujet facile, French Cancan est, de fait, un magnifique hommage à la peinture impressionniste, mais égal ement au cinéma, autant dire, pour les deux cas, au mouvement et au regard.

Le fameux finale au Moulin-Rouge, modelé par un formidable déplacement du spectacle de scène vers le cinéma, referme la boucle et complète la trajectoire circulaire décrite tout au long du film, du cinéma vers la peinture, et du spectacle vers le cinéma. En cela, French Cancan dessine une étonnante parabole. À cet égard, Gilles D eleuze (1985, p. 115-116) écrit fort à propos dans L'I mage temps que «[I]a danse déchainée à la fin de French Cancan n'est pas une ronde, un reflux de la vie dans le circuit, dans la scène de théâtre, comme chez 0 phüls, mais au contraire un galop, une façon dont le théâtre s'ouvre à la vie, se déverse dans la vie, entraînant $\mathrm{N}$ ini dans une eau courante agitée». Cette métaphore de «l'eau courante agitée» convient particulièrement au cancan final. Bien que le philosophe ait relevé ailleurs «le goût de l'eau courante chez Jean Renoir», Gilles D eleuze ne mentionne pas French $C$ ancan quand il tente de comprendre ce que l'école française (Grémillon, Vigo et quelques autres) trouvait d'intéressant dans le motif de l'eau. Pourtant, ce que le philosophe écrit alors appréhende à juste titre la fluidité, le rythme et la pensée traversant French Cancan - dont I'histoire est profondément «pénétrée par le rythme» - , un film qui sans cesse interroge «la limite ou le point de fuite des images-mouvement, des images-moyenne» (D eleuze, 1983, p. 116).

Le 7 avril 1956, avant la première du film à $\mathrm{N}$ ew York où il ne peut se rendre, Jean Renoir écrit quelques lignes qui présentent admirablement bien la ligne directrice de la pensée qui traverse French Cancan. Corroborant l'apparente insignifiance du scénario, Jean Renoir affirme qu'il fait juste du cinématographe, pour s'amuser. Avec plus de sérieux, le réalisateur reconnaît bien que French $C$ ancan lui a permis de réfléchir au mouvement, et non pas aux multiples mouvements de caméra dont il semble dire que le cinéma a fait le tour ${ }^{11}$. Grâce à l'efficacité des mouvements de caméra, une certaine latitude sépare en effet le film du spectacle, à cause des mouvements accordés en retour au 
regard du spectateur de cinéma. Q uand il rédige en 1936 «Style et matière du septième art », Erwin Panofsky considère que c'est la mobilité du regard du spectateur de cinéma qui distingue avant tout le cinéma et le théâtre:

Au théâtre, l'espace est statique, c'est-à-dire que l'espace représenté sur scène ainsi que la relation spatiale du spectateur au spectacle sont fixés une fois pour toutes. Le spectateur ne peut quitter son siège, le décor ne peut changer pendant tout un acte [... ]. Au cinéma, la situation est inversée. Là aussi, le spectateur est rivé à son siège, mais en tant qu'être physique seulement, pas en tant que sujet d'une expérience esthétique. D u point de vue esthétique, en effet, il bouge sans cesse tandis que son regard s'identifie à la caméra qui peut se déplacer constamment en profondeur et dans toutes les directions. Or, si le spectateur est mobile, l'espace qui lui est présenté l'est, parallèlement, tout autant. Ce ne sont pas les corps seuls qui se meuvent dans l'espace mais aussi l'espace même qui se rapproche, s'éloigne, tourne, se dissout et se recristallise à travers le mouvement et la mise au point de la caméra et à travers le montage des différentes prises de vue - sans parler des effets spéciaux: flous, métamorphoses, disparitions, ralentis et accélérés, rétroprojections et autres trucages (Panofsky, 1996, p. 113).

Par la distinction qu'il opère entre les expériences physiques ou esthétiques des spectateurs de théâtre et de cinéma, Erwin Panofsky ouvre une perspective particulièrement prometteuse. Suite à cet article, French Cancan permet sans doute de mieux évaluer, par l'entremise de son personnage principal, dans quelle mesure le cinéma convie son spectateur à vivre une expérience singulière, du moins dissemblable de celle du public des salles de spectacle en direct. À cet égard, la frénésie du cancan final constitue indéniablement l'apothéose d'un film où le mouvement est continuel, tant dans les coulisses que sur la scène. Comme l'a judicieusement écrit Jacques Rivette (1971, p. 266), à propos de «la fièvre panique du cancan final», French Cancan est pensé pour le mouvement: «dans cette furie de filles et de linges, sachons voir l'hymne le plus triomphant que le cinéma ait 
jamais dédié à son âme, le mouvement. Q ui, en déplaçant les lignes, les crée. »

Entièrement pensé pour le mouvement, French Cancan est tout aussi entièrement pensé en plans fixes. Le film n'est pas loin, par son refus de faire étalage de prouesses techniques de la caméra, de s'inscrire en faux contre certaines tendances à la virtuosité dans le cinéma. Comme l'indique l'auteur, l'impact de son film ne provient pas de savants déplacements de la caméra ou de poursuites infernales, mais bien d'un regard sur le mouvement (c'est-à-dire comment faire du cinématographe au milieu des années cinquante). La danse est déchaînée, la caméra presque immobile, rarement en mouvement. Si, effectivement, le mouve ment est l'âme du cinéma (tographe), D anglard est peut-être le guide (spirituel) de French Cancan. Indéniablement, les danses et les chants de French $C$ ancan s'organisent spontanément et naturellement en conformité de style avec le cinéma de Jean Renoir. Pivot de la mise en scène de French $C$ ancan, le regard et les déplacements de $D$ anglard règlent entièrement la mécanique bien huilée du film. De long en large, son œil déplace le regard du spectateur de cinéma. À titre de guide, D anglard n'est pas tout à fait un spectateur - avec ce que le terme implique de passivité - mais plutôt un spectacteur, car il active le regard du spectateur du film.

Pour sentir les rythmes de French Cancan, I'œil du spectateur de cinéma ne doit pas suivre la caméra, presque invariablement statique, mais le regard mouvant du spectateur Danglard. La séquence inaugurale du film est à cet égard une belle entrée en matière. Après le générique, la comédie musicale s'apprête à démarrer après une pause permettant de lire une affiche: «Le Paravent $\mathrm{C}$ hinois. $\mathrm{D}$ anglard présente La Belle Abesse dans ses danses orientales. » D ès cette annonce («D anglard présente»), la séquence dévoile les enjeux profonds du film. L'exiguïté de la salle contraste avec la grandeur du M oulin-Rouge de l'épilogue. $\mathrm{Au}$ Paravent Chinois, les clients sont entassés, les tables et les chaises collées les unes aux autres. Au M oulin-Rouge, les danseuses traversent les murs en carton-pâte, descendent de l'étage au bout d'une corde, courent et gesticulent en tous sens. Au Paravent Chinois, la Belle Abesse peut juste effectuer quelques pas de danse sur une scène misérable. Là, rien ne prête, a priori, 
au mouvement. Pourtant, la mobilité du regard de D anglard va imprimer un rythme certain à la scène inaugurale.

D ans cette première séquence d'une quinzaine de plans, à l'exception de deux ou trois légers panoramiques ou travellings, la caméra est rarement mobile. Le premier plan reprend, presque à la lettre, les mots de Renoir : la Belle Abesse se trémousse, avivant le désir de son public masculin par une danse du ventre qui met en mouvement un corps presque stationnaire. Son corps se balance plus qu'il ne se déplace sur la scène, particulièrement exiguë. Au plan suivant, Danglard fait son entrée en scène dans le film, alors qu'il rejoint un Pierrot siffleur dans les coulisses. D epuis les loges, il croise du regard la Belle Abesse en plein show. Le siffleur lui avoue son trac, et demande à $D$ anglard s'il sera dans la salle pour le soutenir. Le patron du Paravent Chinois pivote sur ses talons, et lui répond pour le rassurer: "T 'en fais pas. Je ne te quitterai pas des yeux. »D anglard s'en va des coulisses pour rejoindre la salle. Au milieu du public, il assiste aux ultimes ronds de jambes de la Belle Abesse. La caméra recule au gré des pas de $D$ anglard, puis effectue un léger mouvement latéral. D anglard tourne maintenant le dos à la caméra qui le suit; il applaudit la sortie de la danseuse, pour applaudir aussitôt l'entrée du siffleur.

Changement de plan : D anglard, face à la caméra, fait un clin d'œil au siffleur, qu'il ne quitte effectivement pas des yeux. II sort du cadre, laissant un instant le champ vide. À une table, un militaire, le Baron Walter et quelques élégants discutent politique. $M$ aintenant que la Belle Abesse n'est plus là, ils tournent le dos au spectacle. D anglard les rejoint, et s'empare d'une chaise qu'il dispose face à la scène. Le siffleur, cadré en légère contreplongée depuis les premiers rangs, poursuit ses pépiements, toujours sous la protection du regard de $D$ anglard, qui est le pre mier à l'applaudir. La Belle Abesse, maintenant en tenue de soirée, se joint aux spectateurs, qui l'ovationnent à son arrivée dans la salle. D 'objet de tous les regards, elle est maintenant à son tour spectatrice. Du moins, rappelle-t-elle que le spectacle peut se déplacer dans la salle. $D$ anglard fait mine de ne pas bouger, les yeux fixés sur le siffleur. Si le mouvement est bien l'âme du cinéma, $D$ anglard est certainement la conscience d'un regard mobile dans F rench Cancan. 
Les mouvements sont entièrement pensés par (pour) le regard de ce spectateur-là. Ce n'est pas par hasard que D anglard dit au Pierrot qu'il ne le quittera pas des yeux. Parmi les spectateurs du Paravent $C$ hinois, Danglard est le seul à accéder aux coulisses, d'où il ne perd rien du spectacle en cours. C'est encore lui qui dirige le spectateur externe et son regard dans ses déplacements vers le Paravent (un objet qui sert à empêcher de voir). C'est derrière $D$ anglard, le seul spectateur mobile du film, que la caméra s'arrête pour ne pas quitter le récital du siffleur. Le groupe qu'il rejoint est assis, tournant le dos à la scène, al ors que lui se déplace constamment et ne rate rien du spectacle. Ce n'est pas par hasard s'il dit à Esther G eorges (Anna Amendola, avec la voix de Cora Vaucaire), alors qu'il échancre sa robe avant son entrée en scène le soir de l'inauguration du M oulin-Rouge: «Là, et n'oublie pas, hein! Chaque type dans la salle doit croire que tu chantes pour lui tout seul. » Enfin, la distinction qu'opère Erwin Panofsky entre les expériences physiques ou esthétiques des spectateurs de théâtre et de cinéma résonne encore davantage quand il s'agit de confronter entre elles deux des dernières séquences de French Cancan : la tournée de $\mathrm{N}$ ini et du Prince Alexandre dans les cafés-concerts parisiens et l'épilogue au M oulin-Rouge.

Lors de leur virée nocturne, $\mathrm{N}$ ini et le Prince Alexandre entrent tour à tour à l'Alcazar d'été, au Chat noir, au Petit Casino et à l'Eldorado pour y écouter quelques-unes des plus grandes vedettes du café-concert, des vedettes comme Yvette Guilbert (Patachou), Paul D elmet (André Claveau), Paulus (Jean Raymond) et Eugénie Buffet (Édith Piaf). Cette séquence est, de fait, bien plus que la fidèle évocation d'une époque et de spectacles en vogue autour de 1900. Au cours des différentes incursions dans les cafés-concerts, la caméra adopte des points de vue chaque fois différents. Pourtant, l'absence de $D$ anglard va se faire vivement sentir. À l'Alcazar d'été, Yvette Guilbert est cadrée de relativement près. De gauche à droite, elle balaie du regard un public qui demeure hors champs, et ce sans jamais s'arrêter face à l'œil de la caméra. Au Chat noir, Paul D elmet chante au milieu des spectateurs, dont font partie $\mathrm{N}$ ini et Alexandre, assis à une table. Au Petit Casino, Paulus est sur une 
estrade, avec derrière lui un décor peint. Alors que $\mathrm{N}$ ini et Alexandre sont à un balcon, le regard du chanteur se déplace de gauche à droite, et se trouve surtout dirigé vers le public assis face à lui au rez-de-chaussée. À I'Eldorado, N ini et Alexandre sont au milieu d'un public disposé en rangs sur des chaises.

Lors des saynètes de French $\mathrm{C}$ ancan, $\mathrm{N}$ ini et Alexandre regardent chaque fois les spectacles d'un angle légèrement différent. $M$ ême s'ils se placent à des endroits différents, d'une salle à l'autre (à un balcon, à une table, dans une rangée de sièges alignés), même si rien ne les empêchent de participer au spectacle, $\mathrm{N}$ ini et Alexandre n'en sont pas moins des spectateurs immobiles. Leur passivité le confirme. En aucun temps, leur regard - dont la liberté de mouvement est restreinte à l'espace de la salle et de la scène - n'ira plus loin ; l'image a pour cadre leur champ de vision. Les séquences au Chat noir et au MoulinRouge disent qu'une règle peut être transgressée par l'artiste de caféconcert: au gré de sa fantaisie, il peut quitter les planches et faire éclater l'espace apparemment confiné de la représentation. Contrairement à celui du thêâtre bourgeois, l'espace des cabarets n'est pas clos. Pourtant, alors que l'artiste conserve au cabaret une certaine liberté de mouvements, c'est uniquement au cinéma, comme l'a judicieusement noté Erwin Panofsky, que le spectateur «est rivé à son siège, mais en tant qu'être physique seulement, pas en tant que sujet d'une expérience esthétique». C'est bien là qu'il y a un écart entre les expériences respectives de $\mathrm{N}$ ini et d'Alexandre d'une part, et de D anglard de l'autre, entre le mode de consommation d'un spectacle de café concert et le mode de consommation d'un film, et, si l'on veut, entre le regard plutôt arrêté de l'un et le regard davantage mobile de l'autre.

D ans l'ivresse générale du M oulin-Rouge, des spectateurs masculins tentent bien d'entrer dans la danse et de participer, de l'intérieur, au spectacle. Ces spectateurs-là sont vivement éconduits, «remis à leur place» par les danseuses, et refoulés vers les côtés de la scène, en dehors de la représentation. La transgression, essentiellement provoquée par la folie passagère qui a envahi le Moulin-Rouge, est de courte durée, et la norme est vite rétablie. D ebout ou assis, ne pouvant que reprendre en chœur les chansons, les spectateurs restent dans leur grande 
majorité là où ils sont. L'irruption de spectateurs dans le cancan final, manifestation du délire ambiant, fait figure d'exception à une règle établie: les spectateurs respectent au cabaret aussi la distance qui les sépare des acteurs. Enfin, mis à part un furtif travelling entre les jambes relevées des danseuses, le finale est essentiellement filmé en plans fixes. Le contraste entre ce nonmouvement de la caméra et le tourbillon des froufrous est évidemment grand. En établissant ce contraste, la caméra ne déroge pas à une règle maintenue tout au long du film.

Tandis que $\mathrm{N}$ ini vient presque buter sur la caméra avant de repartir aussi vite vers le milieu de la scène, les spectateurs restent invariablement cloués sur place. Une dizaine de plans se succè dent sans trêve: pantois, les protagonistes du film (C asimir, Walter, le chef d'orchestre) sont tétanisés par les grands écarts et les va-et-vient des danseuses. Une fois encore, un spectateur fait exception. Resté jusqu'alors assis dans un fauteuil près des loges, d'où il vit le cancan comme s'il était au milieu des danseuses (une accessoiriste le surprend en train de faire les pas de danse), jetant parfois un coup d'œil depuis les coulisses, D anglard se glisse dans la foule en délire. À peine dans la salle, un spectateur lui dit sèchement, sans doute pour lui rappeler qu'au théâtre un spectateur ne se déplace pas et doit rester immobile pour assister à un spectacle: «M onsieur, vous m'empêchez de voir. » Lorsque D anglard, goguenard, se faufile entre les spectateurs du M oulinRouge, c'est bien l'âme du cinéma qui déambule parmi eux.

French Cancan prend fin sur une belle parabole: tandis que la fête bat son plein au M oulin-Rouge, un spectateur sort du cabaret, ivre mort. II titube sur la chaussée, relève son haut-de-forme pour saluer de loin, sur un mode exagérément théâtral, la caméra et le spectateur de cinéma, dont le regard est certaine ment encore étourdi par les mouvements du cancan final.

Université de Liège

\section{NOTES}

1. Eu égard à cette mise en scène et à la place de choix occupée par les chants et la musique dans la programmation, ainsi qu'à l'installation d'une estrade qui offre les 
artistes au regard de tous les clients, les cafés-chantants prennent, aux alentours de 1860 en France, le nom de cafés-concerts. D ésormais, les dients des cafés-chantants sont les spectateurs des cafés-concerts. D ans les cabarets, l'estrade, à l'origine là pour permettre à tous les gens dans la salle de bien voir, a eu cet effet de dresser une barrière entre l'artiste et le public, et d'orienter le regard du spectateur dans une direction architecturalement établie. J'y reviens plus loin à propos d'une séquence particulière de French Cancan (la soirée de $\mathrm{N}$ ini et d'Alex dans les cafés-concerts).

2. D ans un article consacré au «point de vue», Jacques Aumont insiste sur cela, précisément lorsqu'il aborde l'entrecroisement de l'histoire du cinéma et d'autres arts: «Le cinéma, en tant qu'art de la représentation - c'est-à-dire précisément à partir du moment où il se dégage du spectacle, forain ou sédentaire, pour devenir art - , est pris à cette double ou triple histoire: peinture, photo, littérature. 0 n s'étonnera peutêtre de ne pas trouver ici mention du théâtre: c'est que, la suite de ce texte le précisera, le point de vue au cinéma n'a guère à voir avec un "point de vue" théâtral qui est plutôt question d'architecture, et que, d'autre part, l'histoire de la forme filmique je ne dis pas l'histoire du cinéma - n'a pratiquement rien à voir avec celle du théâtre. [... ] Et si I'histoire du cinéma rencontre celle du théâtre, c'est essentiellement par le biais des acteurs - c'est-à-dire sur un plan économique ou sociologique bien plus que sur un plan esthétique» (Aumont, 1983, p. 6 ; note 9 , p. 25). Je renvoie également aux remarques décisives de Jacques Aumont (1996, p. 74-76) concernant l'approche formaliste et la «poétique historique du film » dans A quoi pensent les films.

3. II est frappant de constater que le théâtre est toujours (ou presque) convoqué pour souligner ses effets néfastes sur le cinéma (dont l'histoire néglige l'influence qu'ont pu avoir Brecht, Artaud, Piscator ou Craig, pour ne nommer que ceux-là, sur I'histoire de l'art du $x x^{e}$ siècle). À ce propos, je renvoie le lecteur aux articles d'E ric de Kuyper (dont «Le théâtre comme "mauvais objet" », 1997).

4. Voir mon article «D el mudo al parlante, del café-concierto a la época del jazz» (Arnoldy, 1998, p. 24-37). II va sans dire que j'aurais très bien pu articuler mon propos autour de quelques longs métrages des «premières années du parlant » (T he Jazz Singer, The Singing Fool, M amy, etc.). D ans ces films, là aussi le «passage au parlant » (ou, si l'on veut, le passage de la scène à l'écran, du chanteur à l'acteur) s'effectue à coups de regards signifiants, et sous le couvert d'une mise en scène qui en fait au moins autant pour l'œil que pour l'oreille. J'en ai parlé lors de ma conférence menée à U dine, dans le cadre du colloque consacré aux versions multiples, dont les actes doivent paraître au premier semestre 2003 ( «D e la forme sérielle. L'entre du muet et du parlant: spectacle, chanson, cinéma et Cie»). N éanmoins, je pense que la distinction entre les regards aux spectateurs est d'autant plus criante à travers ces courts métrages Vitaphone, qu'il s'agit là incontestablement - du moins à première vue - de «scènes chantées» assez autonomes, de "spectacles filmés», et non de films de fiction entrecoupés de scènes chantées et dansées.

5. Je souligne: attraction est le mot employé dans la plupart des publicités parues à l'époque. La terminologie usuelle à l'époque est un des innombrables indices de ces ressemblances déplacées dont je parlais plus haut. Dans $\mathrm{H}$ on. Will. H. H ays Welcomes Vitaphone in an Address, W ill H. H ays se présente face à la caméra pour consacrer l'avènement du film sonore. $D$ ans son discours d'introduction, qui a certainement pour double objectif de faire la preuve de la fiabilité d'un système d'enregistrement et de dessiner les contours du cinéma sonore des années trente, W ill H. H ays imagine un cinéma où la musique synchronisée va jouer un rôle inestimable, et des films où la «bonne musique» et l'art lyrique parviendront jusqu'aux hameaux les plus reculés. $D$ ans son allocution, il n'est jamais question de cinéma parlant. Soulignant les vertus pédagogiques du cinéma, il y déclare notamment: «D ésormais, le cinéma a cette faculté unique de diffuser la bonne musique partout dans le pays. D ans le futur, ce 
phénomène va se généraliser, à mesure que le Vitaphone mènera les orchestres symphoniques dans les salles des villes de province. II a été dit que l'art du chanteur ou du musicien était éphémère, qu'il existe le temps fugace de sa création. D ésormais, l'artiste et son art sont immortels. » $\mathrm{O}$ utre le message de W ill $\mathrm{H}$. H ays figure au programme de la première de D on Juan : Tannhaüser (N ew York Philharmonic O rchestra, 17 juin 1926, n 314) ; H umoresque de D vǒrák et Gavotte de Gossec (M ischa Elman et Josef Bonime, 2 juillet 1926, $\left.\mathrm{n}^{\circ} 275\right)$; T heW izard of the Strings, in "H is Pastimes" (Roy Smeck, 14 juillet 1926); Caro Nome extrait du Rigoletto (M arion Talley, 15 juillet 1926, no 308); Kreutzer Sonate de Beethoven (Efrem Zimbalist et $\mathrm{H}$ arold Bauer, 6 juillet 1926, n 279); Vetti La Giubba de Leoncavallo (Giovanni M artinelli, juin 1926, n 198); La Fiesta (Anna Case et les chœurs du M etropolitan O rchestra, 10 juillet 1926, n² 294); et l'O uverture M ignon ( $N$ ew York Philharmonic O rchestra, 29 juin 1926, n²63). Enfin, je profite de l'occasion pour vivement remercier Rick Altman de m'avoir permis de découvrir ces films.

6. Between the Acts at the 0 pera (31 août 1926, no 349); Lambchops (août 1929, n० 891); Twinkle, Twinkle (12 avril 1927, n 505); I dle Chatter (Lou Holtz, 18 décembre 1929). Ainsi, dans des films comme La Fieta ou Behind the Lines, les interventions des spectateurs mettent en exergue tout à la fois la relation d'échange et de complicité qu'une star du music-hall peut avoir avec son public, et la distance qui la sépare du spectateur de cinéma.

7. Gravé et filmé trois semaines après la première de $D$ on Juan, Between the Acts at the 0 pera fait partie du deuxième programme Warner Vitaphone, présenté le 7 octobre 1926 au Colony Theatre de N ew York avant The Better 'O le (de Charles Reisner, avec Syd Chaplin en vedette). Le programme d'ouverture de cette soirée au cours de laquelle est présenté Between the Acts at the 0 pera propose un assortiment de numéros particulièrement respectable, où figurent quelques-unes des plus grandes vedettes de Broadway. $\mathrm{O}$ utre les frères $\mathrm{H}$ oward (grands noms du vaudeville, ils faisaient alors un tabac à Broadway dans Scandals, une comédie de George White), on y retrouve notamment George Jessel, Al Jolson ou Elsie Janis. À cet effet, je renvoie le lecteur aux travaux plus particulièrement consacrés au regard-caméra, à la frontalité spectaculaire et à l'histoire de cette figure dans I'histoire du langage cinématographique (Pascal Bonitzer, Livio Belloï, Francesco Cassetti, André Gaudreault et Tom Gunning, ou encore Christian M etz).

8. D ans son ouvrage sur la comédie musicale hollywoodienne, Rick Altman (1992), que je remercie au passage d'avoir mis à ma disposition certains films dont il a été question ici, y insiste suffisamment, entre autres à propos de Applause de Rouben M amoulian et des «films de coulisses».

9. Après cette allusion à une conférence donnée par Jean Châteauvert et André Gaudreault à Washington à propos des modes de consommation des films (solitaire ou solidaire), j'ajouterai deux mots à propos des Vitaphone shorts où il s'agit bien de montrer combien les spectateurs des salles des vaudeville sont solidaires des vedettes en représentation. Ainsi, dans La Fiesta, dont Variety critiquera la mise en scène (visuelle et sonore), Anna Case parade d'abord pour les danseurs et les figurants qui encerclent la scène. Lorsque d'un geste du bras elle signifie clairement la fin de son chant (et du film), Anna Case effectue là un geste destiné à ceux-ci. Ce «salut diégétique» est donc d'abord adressé aux spectateurs dans le film. Behind the Lines (août 1926, n 339), un film sorti trois semaines après la première de D on Juan en avant programme de Better 'Ole, obéit aux mêmes choix de mise en scène. Elsie Janis est sur une estrade où elle interprète quelques-uns des grands classiques de la chanson populaire américaine ou française ( «W hen Yankee D oodle Learns to Parley vous», «M adeIon», etc.), accompagnée par les chœurs du 107e Régiment. L'étoile du vaudeville chante et blague avec un parterre de soldats. Elle les interpelle, avant d'inviter deux 
militaires à venir chanter à ses côtés. $D$ ans ce film destiné à rendre compte du tour de chant effectué par Elsie Janis sur le front en 14-18 et de la complicité qui la lie à son public, elle ignore superbement le spectateur de cinéma. Elle se donne en spectacle dans une salle où le public applaudit, et dialogue avec la vedette.

10. «Un semblant de cinéma» écrira M itry; «la disparition des travellings achevait de ramener la technique vingt ans en arrière» dira Sadoul, en écho à certaines déclarations de l'époque où des journalistes et des réalisateurs déplorent le retour en grâce d'un cinéma populaire et des spectacles des cabarets. Cela étant, il ne me semble pas nécessaire de dire que cette manière de distinguer le cinéma du théâtre (en gros: cinéma vs théâtre filmé), ou plus exactement le regard de leurs spectateurs respectifs, nous éloigne passablement des débats stériles autour dudit «théâtre filmé» et, plus général ement, des propos caricaturaux à l'encontre d'un théâtre à abattre sans sommation dès qu'il approche les rives du cinéma. Enfin, il ne me parait pas nécessaire de revenir sur les nombreuses comédies musicales du début des années trente, dont les productions de Busby Berkeley, où la caméra est à proprement parler déchainée.

11. Jean Renoir (1974, p. 363) écrit : «Je me sens de plus en plus attiré vers ce genre d'histoires, des histoires assez faibles pour me laisser libre de m'amuser à faire du cinématographe. Sans être très fort en grec, je sais que ça consiste à inscrire des mouvements. Les mouvements que j'aime ne sont pas nécessairement produits par des chevaux galopant ou des automobiles roulant dans des ravins. Les gestes d'une fille arrangeant sa chevelure me suffit, ou la respiration d'une belle femme nue dormant dans son lit, ou un chat qui sétire. $D$ ans French Cancan, j'ai essayé quelques mouvements de ce genre, avec Françoise Arnoul trimbalant son panier à linge, avec les petits marchands de la rue de Paris, avec des danseuses s'exerçant. »

\section{RÉFÉREN CES BIBLIOGRAPHIQUES}

Altman, 1992: Rick Altman, La Comédie musicale hollywoodienne, Paris, Armand Colin, 1992.

Arnoldy, 1998: Édouard Arnoldy, «D el mudo al parlante, del caféconcierto a la época de jazz », Archivos de la Filmoteca, n 30, 1998, p. 24-37.

Anonyme, 1928: Variety, 20 juin 1928.

Aumont, 1983: Jacques Aumont, «Le point de vue», Communications, n 38, 1983, p. 3-29.

Aumont, 1996 : Jacques Aumont, À quoi pensent lesfilms, Paris, Séguier, 1996.

Bazin, 1971 : André Bazin, Jean Renoir, Paris, Champ Libre, 1971.

Deleuze, 1983: Gilles D eleuze, L'I mage mouvement, Paris, M inuit, 1983.

D eleuze, 1985: Gilles D eleuze, L'Imagetemps, Paris, M inuit, 1985.

Kessier et Lenk, 1997 : Frank Kessler et Sabine Lenk, "L'Adresse-M éliès», dans Jacques $M$ althête et $M$ ichel $M$ arie (dir.), Georges $M$ éliès, l'illusionniste fin de siècle?, Paris, Presses de la Sorbonne N ouvelle, Colloque de Cerisy, 1997, p. 183-199.

Kuyper, 1997: Eric de Kuyper, "Le théâtre comme "mauvais objet" », Cinémathèque, no 11, 1997, p. 63-75.

Panofsky, 1996 : Erwin Panofsky, «Style et matière du septième art », dans Trois Essais sur le syle, Paris, Gallimard, 1996.

Renoir, 1974 : Jean Renoir, Écrits، 1926-1971, Paris, Belfond, 1974.

Rivette, 1971: Jacques Rivette, «French Cancan», dans André Bazin, Jean Renoir, Paris, Champ Libre, 1971. 